\title{
LAS REPRESENTACIONES SOCIALES DE LA PARTICIPACIÓN POLÍTICA DE LAS MUJERES LIDERESAS DE LA CIUDAD DE MEDELLÍN. ANÁLISIS DESDE EL ENFOQUE INTERSECCIONAL
}

\author{
Nataly Restrepo Restrepo \\ nrestr13@tdea.edu.co \\ Tecnológico de Antioquia - Colombia
}

Dora Isabel Aristizabal Hoyos

doraisabelaa@yahoo.com

Sec.de Educación de Medellín - Colombia

\author{
Natalia Guerra Lopera \\ natalia.guerra@uniminuto.edu \\ Centro de Educación para el Desarrollo - Colombia
}

\author{
Gladys Rocio Ariza Sosa \\ gariza@ces.edu.co \\ Universidad CES - Colombia
}

Recibido: 29-02-2016

Aceptado: 11-05-2016

\section{Resumen}

Los procesos de participación política de las mujeres lideresas populares en Medellín en el siglo XXI han estado mediados por las representaciones sociales hegemónicas y patriarcales que se han tejido alrededor de los roles y estereotipos asignados socialmente a las mujeres. Los resultados de esta investigación de corte cualitativo señalan que para ellas la representación social de la participación política está asociada a su presencia en los diferentes espacios en los cuales pueden opinar para tomar decisiones e incidir en beneficio de la comunidad, no obstante ello se ve obstaculizado por las diferencias de género, en articulación con otras categorías interseccionales como: la etnia, la diversidad sexual, la discapacidad, la edad o el ser víctima del conflicto armado, entre otras. Por tanto, las mujeres sugieren propuestas encaminadas a favorecer desde la primera infancia los procesos de formación, así como políticas públicas enfocadas a la sororidad y el trabajo en red, así como a la transformación de las feminidades y las masculinidades hegemónicas.

Palabras Clave: Participación política, mujeres, interacción social, liderazgo político, interseccionalidad.

\begin{abstract}
The processes of political participation of popular women leaders in Medellin in the XXI century have been mediated by the hegemonic and patriarchal social representations that have been hatched around the roles and stereotypes assigned to women in society. The results of this qualitative research show that, for them, the social representation of political participation is associated with their presence in different spaces in which they can influence to make decisions for the benefit of the community, however hampered by gender differences in linkage with other intersectional categories such as ethnicity, sexual diversity, disability, age or being a victim of armed conflict, among others. Therefore, women suggest proposals to promote from early childhood training processes and public policies focused on sisterhood and networking, as well as the transformation of femininities and hegemonic masculinities.
\end{abstract}

Keywords: Political participation, women, social interaction, political leadership, intersectionality. 


\section{Introducción}

Desde la segunda mitad del siglo XX, la ciudad colombiana de Medellín se ha caracterizado por ser un territorio con una gran capacidad de organización y movilización ciudadana evidenciada en la cantidad de organizaciones comunitarias que la habitan, en el número de movimientos sociales diversos, en sus reivindicaciones y en la presencia de formación y organización que se promueve desde las bases de procesos sociales, que involucran a diferentes sectores de la población como las organizaciones de mujeres, jóvenes, estudiantes, ambientalistas, gremios y adultos mayores (Jurado, 2003).

Este contexto ha motivado la construcción desde distintas expresiones a nivel estatal y gubernamental, de espacios para el debate, la confrontación, la negociación y la toma de decisiones para enfrentar las múltiples problemáticas que aquejan los barrios de la capital antioqueña, y, además, para promover procesos de desarrollo local contextualizados, sostenibles y equitativos. El surgimiento de las organizaciones sociales ha contribuido a mitigar los procesos de socialización patriarcal y por ende su reproducción, siendo éstos aún insuficientes (Tello, 2009).

La familia, la escuela y la Iglesia católica, ya no son las instancias hegemónicas de socialización de las generaciones jóvenes; las nuevas prácticas y sociedades de pares (grupos juveniles, barras, "parches", "combos", pandillas, galladas), han tomado un importante protagonismo y autonomía en la socialización juvenil y en el surgimiento de valores y actitudes que a veces contradicen los de la sociedad adulta tradicional (Jurado, 2003).

Es precisamente en estas distintas expresiones de escenarios locales de la participación política de la ciudadanía en Medellín donde se evidencian, las limitaciones de la incidencia de las mujeres frente al tradicional control de los hombres. Diversas investigaciones exponen como las mujeres han generado procesos de resistencia creando organizaciones sociales y promoviendo movilizaciones ciudadanas para reivindicar sus derechos, sin embargo, en algunos espacios siguen siendo excluidas con más fuerza (Tello, 2009; Atehortua y Rojas, 2005; Luna y Villareal, 1994).

Durante el siglo XX las mujeres en Colombia, han ganado un mayor reconocimiento, gracias a las múltiples acciones realizadas en los diversos escenarios (educativo, social, político) no obstante, aún se requieren cambios profundos en el tejido social que contribuyan a socavar las inequidades bajo las cuales, ha estado supeditada la vida de las mujeres, por supuesto con variaciones históricas y geográficas (Silva, 2008).

El poder otorgado al mundo masculino (patriarcal), se ha mostrado como natural y ahistórico, como un privilegio "divino" y proveniente de un "orden natural" para justificar la opresión ejercida contra las mujeres (Cobo, 2005), y es por ello, que no es en vano que la relación entre hombres y mujeres haya estado marcada tradicionalmente por numerosas 
diferencias que se muestran como derivadas del seno de lo biológico, para trasladarse al incesante mundo de las desigualdades sociales. No obstante, las mujeres no se supeditaron al abismo de la exclusión y han generado procesos de lucha, resistencia y visibilización que en el siglo XXI les ha permitido gozar de derechos, nunca antes imaginados.

De este modo se deduce que los obstáculos al acceso a los escenarios de la democracia participativa en las comunas de Medellín encontrados por las mujeres, pasan precisamente por este poder patriarcal. El trabajo reproductivo de las mujeres ha sido utilizado como una barrera naturalizada para limitar su participación en los espacios políticos y públicos.

Estos escollos son parte de una herencia histórica derivada de la imposición de innumerables valores que pretenden restringirlas a una condición artificiosa de pureza y virginidad, como la del modelo del "ángel del hogar" difundida ampliamente en Hispanoamérica entre las décadas de los años treinta a cincuenta del siglo XX (Luna, 2002). Según Puyana y Mosquera (2005) la tendencia tradicional acerca de la corporalidad de la mujer corresponde a una imagen de la maternidad: servicial, pasiva, emocional, que ama sin límites. Y por su parte, el cuerpo masculino: estereotipado como el activo, creativo, racional, viril y proveedor. En nuestra cultura la representación ha sido mujer $=$ madre, hombre $=$ proveedor, y es por ello que, en esta concepción, se considera que el hijo pertenece más a la madre.

Desde esta perspectiva, se evidencia como la maternidad ha sido un encargo para las mujeres, lo cual genera que, en los procesos de socialización, se les eduque para permanecer más en el ámbito privado, y a los hombres por el contrario se les estimula a participar al ámbito público. Así, las mujeres colombianas como lo señala Idrovo (2006) se ven obligadas a conciliar la vida privada con la vida pública, haciendo de esta situación, una tarea titánica para muchas de ellas, dada la cantidad de responsabilidades que tienen en el hogar y las limitaciones que encuentran en sus ámbitos de trabajo, que se encuentran no sólo ligados al empleo formal, sino también a la informalidad.

En este sentido, aunque las mujeres han logrado incorporarse (insuficientemente) en las instancias públicas, su participación se reconoce por la lucha y organización en los movimientos sociales donde han logrado su reconocimiento como sujetas de derecho, y como agentes activos en el desarrollo económico y social de Colombia. No obstante, aún no se consolidan plenamente las garantías que les permitan ubicarse como protagonistas en el escenario político en igualdad de oportunidades que los hombres (Tello, 2009).

A pesar de que se ha avanzado de manera significativa en el reconocimiento social y político de las mujeres, siguen existiendo cuestionamientos frente a su representatividad en los escenarios políticos del país. Se han promulgado leyes tendientes a cerrar esta brecha como la denominada Ley de cuotas 581, (Colombia, Congreso, 2000) que exige al menos un $30 \%$ de mujeres en los cargos públicos, pero su implementación aun presenta notorias dificultades que impiden hablar de una igualdad real en la materia (Andrade, 2006). 
Las representaciones sociales hegemónicas sobre las relaciones de género han generado obstáculos para la participación plena de las mujeres en los escenarios políticos, por supuesto con matices dados por la interseccionalidad con categorías como la clase social, la etnia, la orientación sexual o la situación de discapacidad, entre otras (Expósito, 2012).

La teoría de las representaciones sociales permite esclarecer cómo la intersección de diversas características o identidades de las mujeres, les afecta la posibilidad de acceder a los escenarios democráticos de la vida política local, y poder así, contribuir efectivamente en el fortalecimiento del ejercicio gubernamental y social de la ciudad de Medellín, a partir de la garantía irrestricta de sus derechos políticos.

Con el propósito de aportar desde el saber académico de las ciencias sociales a la discusión social entorno a la escasa participación e incidencia de las mujeres en los escenarios de participación política de ciudades como Medellín, se planteó la siguiente pregunta de investigación ${ }^{1}$ :

¿Cuáles son las representaciones sociales de la participación política de las mujeres lideresas de la ciudad de Medellín? Ello con el género como categoría analítica central y desde el enfoque interseccional.

El objetivo de esta investigación es comprender las representaciones sociales de la participación política de las mujeres lideresas de la ciudad de Medellín, a partir del enfoque interseccional

\section{Metodología}

Esta investigación se realizó a partir de un enfoque hermenéutico (Creswell, 2003). El proceso de comprensión de las representaciones emprendido es de tipo circular, ya que las investigadoras a partir de los criterios de validez y confiabilidad, consultaron permanentemente el problema y la interpretación de los datos recopilados.

Se realizó muestreo intencional, de acuerdo a las características establecidas según los objetivos y se eligieron las mujeres que se consideraban que compartían determinadas características de interseccionalidad establecidas. Este tipo de muestreo facilita la posterior transferibilidad de los resultados de la investigación (Guba, 2008). El principio para establecer la cantidad de participantes a entrevistar fue el de saturación teórica de las categorías (Corbin y Strauss, 2002).

Se entrevistaron 14 mujeres con edades entre los 23 y 56 años, quienes residen en la ciudad de Medellín en cinco comunas urbanas, y dos corregimientos rurales, sus viviendas

\footnotetext{
${ }^{1}$ Esta investigación se desarrolló en la Institución Universitaria Tecnológico de Antioquia de Medellín, como trabajo de grado de la maestría en educación con énfasis en poblaciones vulnerables.
} 
están ubicadas en los estratos 0 a 6 , es decir se entrevistaron mujeres de todas las condiciones socioeconómicas. Su origen es diverso, algunas son oriundas de esta misma ciudad y otras fueron desplazadas desde el campo por la violencia o emigraron hacia Medellín en la búsqueda de una mejor calidad de vida. A nivel de escolaridad, algunas tienen la básica primaria, otras cursaron sólo hasta noveno grado, las demás cursaron formación técnica, están terminando el bachillerato o son profesionales.

Para la recolección de datos, la técnica elegida fue la entrevista a profundidad. La guía se estructuró en cuatro partes, para caracterizar a las mujeres entrevistadas, obtener información sobre la configuración, contenidos, prácticas y dinámicas de las representaciones sociales de la participación política, así como indagar por las recomendaciones de las participantes para promover su participación política.

Se utilizaron los procedimientos de la teoría fundada como metodología (Corbin y Strauss, 2002) para establecer las categorías a partir de la recolección de datos en las entrevistas. Es importante precisar que la teoría fundada se empleó como estrategia metodológica, no como teoría en sí, en atención a que ello significaría llegar al campo en blanco y las investigadoras partieron de un referente teórico feminista (Lagarde y De los Ríos, 2003) en articulación con la teoría de las representaciones sociales en su enfoque procesual (Jodelet, 1988).

El análisis se constituyó a partir de procedimientos de codificación abierta, axial y selectiva de las cuales emergieron códigos de análisis, de acuerdo con las características comunes, y a partir de la frecuencia y densidad, se dio paso a la organización de las familias, según las relaciones entre los códigos. Posteriormente se construyeron los mapas conceptuales, y de allí se inició el proceso de consolidación de conceptos y de la teoría que emergió del discurso de las mujeres, con un estricto manejo de la información.

\section{Resultados}

Los resultados dan cuenta de las acciones personales, sociales y políticas que las mujeres pueden ejercer, para acceder al ejercicio de liderazgo político en la ciudad de Medellín. Estos hallazgos, construidos desde las voces de las mujeres entrevistadas, las ubican como protagonistas a partir de sus relatos, experiencias, aprendizajes y objeciones.

Se resaltan, además, sus propuestas, las cuales pueden contribuir a repensar nuevas propuestas de ciudad, no sólo en Medellín, sino en otros escenarios similares, que impacten en el ejercicio de los derechos y la participación política de las mujeres. 


\section{Figura 1. Categorías emergentes de la investigación}

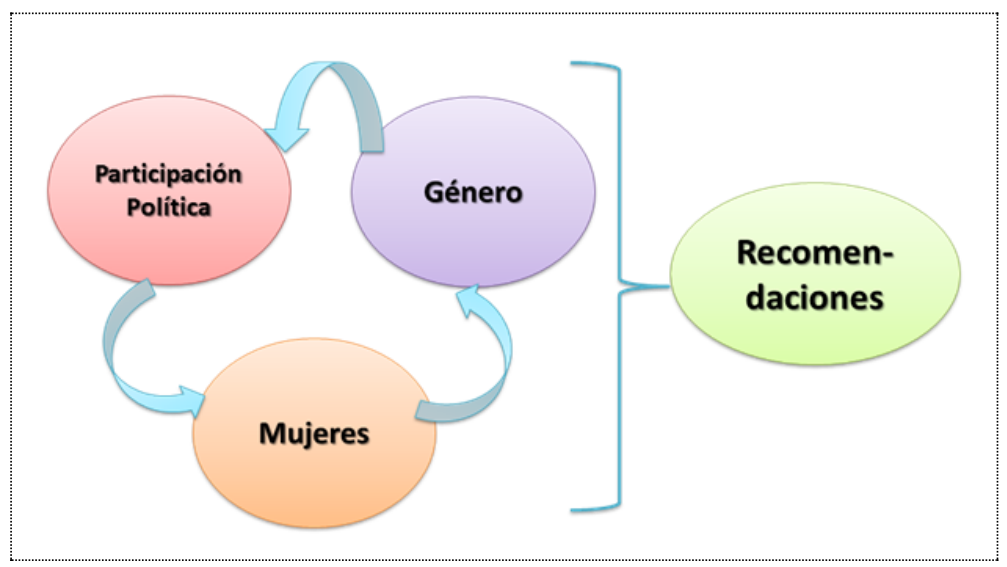

Fuente: Elaboración propia de las autoras basada en los hallazgos de esta investigación

La figura 1 muestra las cuatro categorías principales que describen los resultados a partir de la organización, codificación, y el análisis de la información. Las representaciones sociales de las mujeres entrevistadas, respecto a su ejercicio político en sus territorios se pueden observar en las categorías mujeres, género y participación política. Las recomendaciones emergen del planteamiento de propuestas, estrategias e iniciativas que permitan al orden gubernamental cualificar las estrategias que potencien la participación política de las mujeres en la ciudad de Medellín.

Las mujeres configuran la representación social de su participación política, en los procesos de interacción y comunicación que establecen con los sujetos con los que se relacionan cotidianamente. Cuando ejercen su proceso de liderazgo, construyen y estructuran nuevas subjetividades, a partir de sus propias historias de vida, generando nuevos aprendizajes, mediante un intercambio de información y contenidos que transforman sus estructuras de pensamiento y determinan sus formas de ser y actuar en el contexto. Como señaló una de ellas “ya no somos las mismas” (E2).

El contenido de sus representaciones sociales da cuenta de las construcciones colectivas que se han tejido en los diversos procesos en los que participan. Por ello, para este grupo de mujeres se estructuran conceptos en relación a la participación, el empoderamiento, el liderazgo, la incidencia, el género y la interseccionalidad.

Ellas consideran que la participación política se relaciona con su presencia en los diferentes espacios en los cuales pueden opinar para tomar decisiones e incidir en beneficio de la comunidad. Esta participación se facilita gracias al empoderamiento que han adquirido en los procesos de formación y trabajo comunitario en donde han logrado reconocimiento de sus derechos, transformar sus formas de pensar, perder el miedo para opinar y tomar decisiones. Todo esto les permite incidir y liderar diversos procesos constructivos para sus territorios. 
Las representaciones sociales en relación al género se han ido modificando de acuerdo a las experiencias y procesos formativos adquiridos por las mujeres en el trasegar de esta labor. Sin embargo, estos aprendizajes no han sido suficientes para superar las barreras que emergen cuando se trata de participar. Las mujeres enuncian que en los procesos de participación se evidencian diferencias profundas entre hombres y mujeres relacionadas con el género y la desigualdad que se ve reflejada en estos escenarios. Aunque en ocasiones son más las mujeres, los hombres siguen siendo más visibilizados y ostentan el poder. Ellos subvaloran la participación de las mujeres en espacios en los que tienen notorio peso las estructuras simbólicas de la cultura patriarcal que les impiden a ellas el ejercicio pleno de su derecho a participar, lo cual se puede considerar entonces como parte de la violencia estructural.

Además, en sus declaraciones se identificaron algunas representaciones sociales hegemónicas, relacionadas con las construcciones culturales donde refieren que el poder lo han tenido tradicionalmente los hombres, y que, en este sentido, se supone que las mujeres deberían ocuparse del ámbito privado y los hombres del público. Por su parte las representaciones sociales emancipadas que se identificaron en sus relatos se refieren al tema de la sororidad y la posibilidad de la validación y el reconocimiento de las otras mujeres; así como a las diferencias en la participación con relación a las categorías interseccionales.

Las representaciones polémicas significan formas de pensamiento diversas y contrarias a las predominantes, por lo que se ubicaron en éstas, algunas creencias sobre la ausencia de una cultura machista en sus escenarios de labor comunitaria y que en consecuencia todas las mujeres participan en igualdad de condiciones con los hombres y entre sí, es decir la interpretación de que ni el género ni la interseccionalidad afectan dichos procesos.

\subsection{Mujeres diversas líderes y empoderadas}

En la categoría de mujeres, a partir de la información recolectada, emergieron elementos relacionados con: empoderamiento, liderazgo, incidencia, logros obtenidos, formación, derechos de las mujeres, transformación personal y cultural, motivación, contexto social.

Como ya se mencionó se entrevistaron mujeres adultas, tanto de origen urbano como rural, de diferentes condiciones socioeconómicas y grados de escolaridad desde la primaria hasta la universidad.

Desde el punto de vista de la interseccionalidad, entendida ésta, como la comprensión de las múltiples identidades y maneras de exclusión, que se expresan en opresiones, las mujeres entrevistadas se caracterizan de manera diferencial por ubicarse bien sea como campesinas, afro-descendientes, lesbianas, en situación de discapacidad, víctimas del conflicto social, en situación de pobreza, jefas de hogar o jóvenes. Algunas de ellas tenían varias de estas características a la vez y todas hacen parte de diferentes escenarios de participación de la ciudad. 
En el momento de las entrevistas declararon variedad de ocupaciones entre las que se resaltan ama de casa, el trabajo informal, el estudio, el trabajo informal independiente y la mayor parte de su tiempo lo dedican a la participación política en los escenarios locales como: juntas de acción comunal, juntas administradoras locales, presupuesto participativo, organizaciones comunitarias, movimientos sociales, entre otros. El liderazgo fue resaltado como su ocupación principal.

Los escenarios más destacados en el inicio de la participación de estas mujeres fueron locales: el colectivo de mujeres, las organizaciones campesinas, las asociaciones de padres de familia, los colegios y las juntas de acción comunal, en diversos cargos.

Respecto a las teorías emergidas en relación con el género, se resalta el empoderamiento como la principal consecuencia de la participación política de las mujeres, lo que lo sitúa como uno de los principales facilitadores para la incidencia en los contextos en los que se desenvuelven. Una de las participantes manifestó: “cuando uno adquiere el conocimiento y se empodera de él, bueno es que yo sé esto y no es lo que el otro diga sino lo que yo sé” (E3).

Las mujeres entrevistadas refieren la importancia de la formación para lograr un empoderamiento efectivo, ya que, en estos procesos, ellas adquirieron aprendizajes que se relacionan con su crecimiento personal, político y social, desarrollaron autonomía y lograron reconocer sus propias capacidades y habilidades para liderar los diferentes procesos. Como manifestaron varias de ellas:

"estando en el colectivo ya pude tener capacitaciones a nivel de la Secretaria de la mujer, a nivel de los proyectos que han sacado por PP (Presupuesto Participativo) para las mujeres, en esos procesos me he empoderado mucho para la cuestión política y social" (Diana E 11).

"se pierde el miedo para pelear lanzar proyectos y defender la comuna" (E2).

Las motivaciones principales de las mujeres cuando iniciaron su participación en los diferentes escenarios se relacionan con una pasión que les nació desde su niñez por el trabajo comunitario y social. Rosa una mujer de 56 años expresa: "desde pequeña me ha gustado la parte social, o sea lo mío viene desde pequeña, toda la vida me ha gustado” (E3). En este orden de ideas, otras mujeres refieren que sus motivaciones para empezar a trabajar por sus comunidades se ligaron con el contexto de pobreza, la violencia, la vulneración de los derechos, las necesidades, los problemas sociales y el sufrimiento de las personas. Amparo comentó: “Cuando uno es líder a uno le nace, no lo obligan” (E2).

Así pues, el empoderamiento ha permitido logros; uno de los más significativos para ellas es el reconocimiento que hace la comunidad a su labor o trabajo y la confianza en su desempeño. Alicia una mujer 43 años comentó al respecto, 
"se gestionan proyectos para la comunidad que benefician a la comunidad, entonces eso también da como reconocimiento al trabajo que uno hace y también da satisfacción saber que uno gestiona para el desarrollo de la comunidad, por eso soy feliz cuando los proyectos salen, y lo disfrute la comunidad" (E1).

Así el empoderamiento les permitió aprendizajes, las mujeres entrevistadas en los diferentes procesos de participación lograron perder el miedo, tomar decisiones, liderar, ser autónomas, identificar sus capacidades y adquirir conocimientos. Así mismo los escenarios de participación se han convertido para ellas en una escuela, donde empezaron a reconocerse, valorarse e identificar sus derechos, respetar al otro, aceptar las diferencias y discriminar los tipos de política. Con relación a lo anterior Karen enunció "personalmente ha sido una escuela de mucho aprendizaje, de mucha experiencia para un asunto pues personal, ya uno aprende también como a conocer los liderazgos, a no desgastarse" (E8).

No obstante narran lo difícil que es mantenerse en estos espacios por las barreras patriarcales que interfieren en su liderazgo, Karen una joven mujer refirió:

"es un proceso de liderazgo en el que hay que empezar a ganar mucho terreno bajo muchas circunstancias y bajo muchas condiciones, ya sea porque los hombres son los que mandan o porque son los que tienen la vocería en muchas cosas, es un espacio que nos venimos digamos ganando, no ha sido fácil porque en la medida que vamos avanzando pues nos vamos adentrando como a ganar más espacios, porque si nosotras nos quedamos ahí como mujeres o retrocedemos es permitir que ellos continúen en cierta forma o en cierta medida liderando estos espacios"(E8).

\section{Figura 2. Categoría: Mujeres}

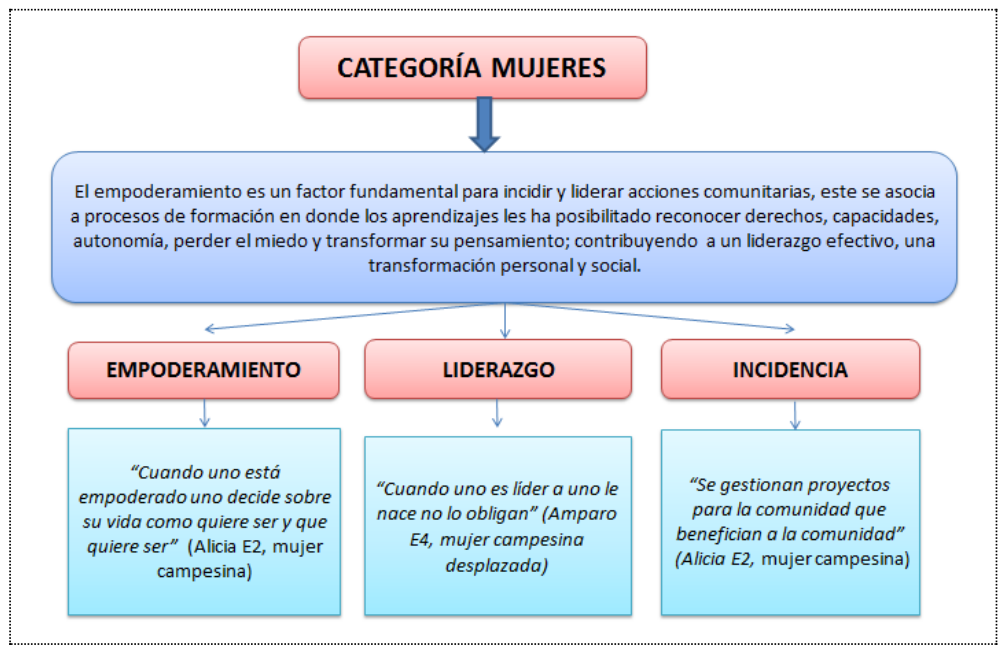

Fuente: Elaboración propia de las autoras basada en los hallazgos de esta investigación 
La figura 2 recoge los tres principales conceptos emergentes en las representaciones sociales de las entrevistadas que se agrupan en la categoría mujeres, esto significa que ellas se definen como líderes empoderadas que inciden en acciones comunitarias. Vale la pena resaltar, además, que esta categoría está relacionada con las características de las mujeres participantes de la investigación y las consecuencias que tiene el ejercicio del liderazgo en sus comunidades, a nivel personal, familiar y social.

\subsection{Desde que me levanto hasta que me acuesto, yo vivo la política}

El análisis de las representaciones sociales de la participación política de las mujeres lideresas de la ciudad de Medellín, constituyó la esencia de esta investigación, ya que se considera profundamente valioso ahondar en las construcciones culturales que han ejercido una fuerte influencia en el rol social tradicional asignado a las mujeres y que evidentemente las ha puesto en una posición de desventaja respecto a los hombres, situación más notoria en los espacios de poder y decisión. Para estas mujeres, su condición sexual conectada con otras identidades se constituye en vulnerabilidad. La etnia, la diversidad sexual, la discapacidad, el conflicto o el empobrecimiento contribuyen a la exclusión y marginación social de gran parte de los ámbitos de la vida pública. Pero por supuesto ellas han emprendido una serie de procesos de resistencia para sobrepasar todo esto.

De esta manera, la categoría participación política está referida a las características, los desencuentros, la transformación de los roles y estereotipos sexistas, frente a los obstáculos y facilitadores encontrados por las mujeres, en su ejercicio de participación política.

Para las mujeres, la participación política está ligada a la posibilidad de incidir en decisiones que contribuyan al beneficio de la comunidad. Este aspecto es muy importante, ya que dicha categoría se ha entendido históricamente de forma predominante desde la representatividad democrática, la cual se materializa a través de los diferentes mecanismos de elección y no en un sentido amplio, como lo es aquella que se refiere a la creación de dispositivos sociales que permiten la deliberación y la intervención en la toma de decisiones de las comunidades, como en este caso, para las mujeres lideresas. Como lo expresa Diana, una mujer habitante de la ruralidad de Medellín: "la participación son los espacios donde uno puede, tiene la palabra opina y dice que quiere pues que haya en la comunidad" (E1).

Las mujeres entrevistadas reconocieron una estrecha relación entre la participación política y el poder. Ana, una mujer sexualmente diversa (lesbiana), expuso que la participación es: "poder decidir y poder participar, si no lo haces tenés que asumir lo que otros están haciendo y aceptar esas decisiones" (E6).

La participación es, vista de esta manera, no como un derecho, sino como un privilegio, al cual pocas personas tienen la posibilidad de acceder; lo que genera como consecuencia, que quienes no porten esta prerrogativa, se ven obligadas a asumir las decisiones de una pequeña colectividad, que afectarán directamente a toda la comunidad. 
En este sentido, participar en un escenario de decisión y pertenecer a la comunidad LGBTI, conlleva a que éstas mujeres traten de exhibir con mayor ahínco, sus habilidades para representar a una comunidad en un escenario político, ya que al no optar por la heteronormatividad predominantemente, les implica asumir las consecuencias de la exclusión, el desagrado y la desconfianza, lo cual tiene como significado no portar "poder".

Respecto a las características de la participación, las mujeres entrevistadas plantearon su representación social a partir del siguiente concepto que se construyó desde las visiones de todas ellas:

"Exponen que ser líder es una cualidad con la que se nace; es una labor que se desea y que hace por gusto, la cual implica creer en las capacidades propias y contar con valores como el respeto por la comunidad, el cumplimiento, la capacidad de convivir con los demás, y el ser asertivas frente a las propuestas y opiniones; para participar de las decisiones que aportan y contribuyen a la comunidad, a través de diversas iniciativas que generan beneficios y las cuales a su vez implican un reconocimiento por parte de la sociedad, a quien lidera".

Amparo, una mujer víctima del conflicto armado, campesina de Medellín, expresó respecto de las características del liderazgo, que para ella: "cuando un líder se queda bastante tiempo es porque le gusta ser lider, uno saber ser lider y saber respetar, sabe ser cumplida” (E2). Lo cual indica que la duración del ejercicio del liderazgo es una clave que determina la capacidad de las mujeres para mantenerse en la esfera pública, a pesar de tener que conciliarla con su vida privada y de cuidado familiar, con todas las consecuencias que esta decisión conlleva.

En relación al liderazgo femenino, las mujeres nombran el reconocimiento como primer legitimador de esta labor. De esto habló Amparo cuando comentó "No es que yo me esté creyendo de nada...por eso estoy ahí, porque por ejemplo yo salgo en los periódicos” (E2). Así pues, para las mujeres contar con el respaldo y la aprobación de la comunidad, es una necesidad insoslayable, ya que hacer uso o pertenecer a escenarios tradicionalmente vetados para ellas, convierte en un verdadero reto el poder participar en éstos y ejercer con plena libertad la posibilidad de incidir y tomar decisiones, sin que estén mediados por nuevos obstáculos.

Debido a los conflictos que emergen en este ejercicio de liderazgo femenino, las entrevistadas señalan que constantemente se presentan entre las mujeres lideresas diferencias que se convierten en problemas de rivalidad frente a su labor. Al respecto Ana nos explicó:

"las mujeres posiblemente no tenían, aun no tienen esa conciencia de que las mujeres tenemos que diferenciar esas necesidades entre hombre y mujeres y no atacarnos, aún hay esa... digamos yo no sé, ese ataque, y aún muchas lideresas no entienden que tenemos que acompañarnos, no conocemos o no conocen el término de sororidad y nos atacamos" (E6). 
El ejercicio político sin alianzas, se convierte en una actividad nula, en el entendido de que no permite incidir efectivamente en el logro de las metas trazadas. En este sentido, se hace necesario resaltar cómo el patriarcado, materializado en las diferentes instituciones sociales, ha promovido históricamente la rivalidad entre las mujeres, perpetuando ideas relacionadas con la enemistad y los conflictos (Lagarde y de los Ríos, 2003), lo cual ha imposibilitado que fluya una alianza fuerte y consolidada, que les permita a estas mujeres concretar mucho más su ejercicio de liderazgo en proyectos y acciones efectivas para la ciudad de Medellín.

Figura 3. Categoría: Participación política

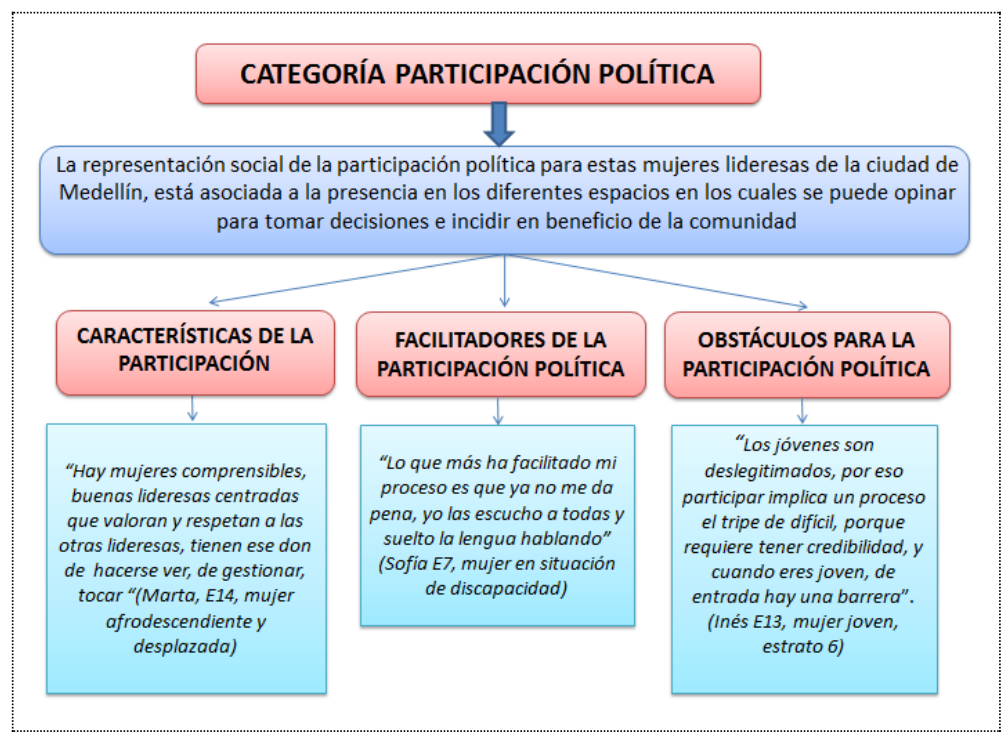

Fuente: Elaboración propia de las autoras basada en los hallazgos de esta investigación

La figura 3 revela el significado general frente al concepto de participación política, que emerge del discurso de las entrevistadas, así como los conceptos vinculados a éste, relacionados con las características, facilitadores y obstáculos de la participación política de las mujeres lideresas de la ciudad de Medellín.

Cabe señalar, que esta categoría permite identificar cuáles son las principales problemáticas a las que se ven enfrentadas las mujeres de Medellín cuando desean acceder al ejercicio de la participación política en sus barrios y comunas; y así mismo, deja ver, como la familia, se convierte en un referente principal de apoyo y complicidad del proceso de liderazgo político de las mujeres. 


\subsection{La mujer la más sentida, la más dolida}

En la categoría género, a partir de la información recolectada, emergieron elementos relacionados con: la diferencia en la participación de hombres y mujeres, el patriarcado y la interseccionalidad.

A partir de las diferencias de género las mujeres se han sentido vulneradas por una cultura de orden patriarcal, dado que no se les ha permitido ejercer la participación en igualdad de condiciones con ellos. Los hombres tienen la posibilidad de acceder a los escenarios políticos comunitarios con mayor facilidad ya que socialmente es un espacio que tienen ganado, mientras las mujeres tienen que luchar para conseguirlo y esforzarse en mayor medida para mantenerse en éstos. Así Diana expresó “a veces las mujeres participan más que los hombres, nosotras las mujeres peleamos eso, nosotras las mujeres participamos más y tiramos más proyectos, pero los hombres siempre ganan” (E11).

Los obstáculos para acceder a los escenarios de participación pueden analizarse a partir de la intersección de categorías analíticas ya que estas mujeres, además de sus características de género se ven vulneradas por otra serie de condiciones como ser campesinas, afrodescendientes, lesbianas, en situación de discapacidad, víctimas del conflicto social, en situación de pobreza, jefas de hogar y mujeres jóvenes. En este mismo sentido Julia expresó:

"Una persona afrodescendiente se tiene que esforzar el doble para que la escuchen que una persona mestiza, digamos que eso es un obstáculo a la hora de intervenir con la gente, como esta negra que me va a decir, uno tiene que esforzarse muchísimo, trabajarles a otras cosas para que la gente como que te escuche y tenga como un respeto” (E4).

\section{Figura 4. Cruce de Interseccionalidades de las Mujeres participantes de la investigación}

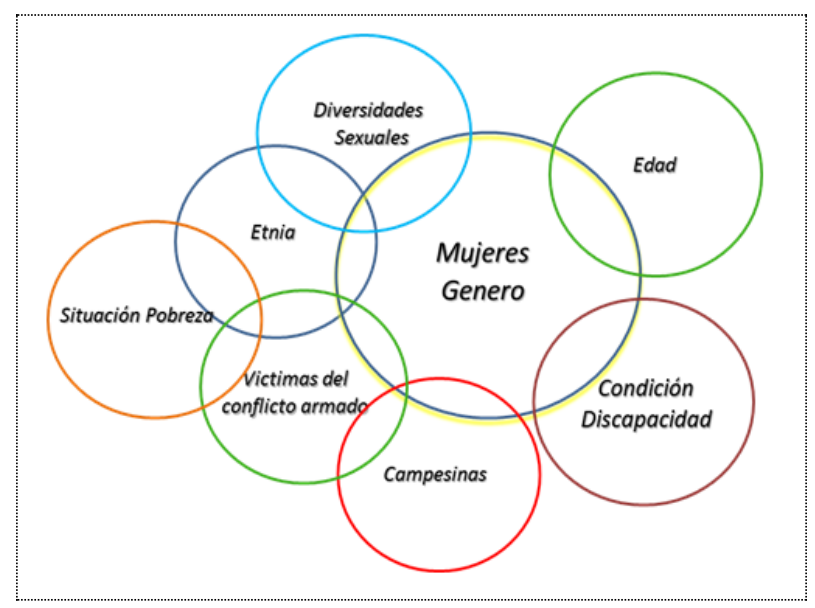

Fuente: Elaboración propia de las autoras basada en los hallazgos de esta investigación 
La figura 4 muestra en forma de teoría de conjuntos la relación de intersección entre las identidades o características de vulnerabilidad de las mujeres entrevistadas, reconocidas e identificadas por sí mismas. Permite revelar, como una misma mujer reconoce en sí misma distintas situaciones de vulnerabilidad que la pueden hacer, en la mayoría de los casos, más propensa a condiciones de exclusión y discriminación de los escenarios de participación política en la ciudad de Medellín. Por ejemplo, se entrevistaron mujeres que se reconocían simultáneamente como afro, pobres y víctimas del conflicto armado, lo cual les dificultaba sus procesos de ejercicio político.

En sus comunidades, ellas identifican una subvaloración de género con relación a su participación. Amparo refiere:

"[...] como que no le dan el mismo valor a la mujer como se lo dan al hombre" (E2). De igual forma Marta una mujer afrodescendiente mencionó: “A pesar de que muchas mujeres nos estamos preparando para estar en lo público, falta credibilidad, porque los hombres siempre nos van a ver 'pordebajiadas' (sic) y que no tenemos capacidad, pero las mujeres tienen esa desconfianza, eso es comprensible porque hemos vivido en un país machista, donde no hay credibilidad en lo que puede hacer la mujer" (E14).

El poder lo han concentrado los hombres, quienes en su mayoría han asumido el liderazgo de los diferentes escenarios en los que ellas se desenvuelven. De igual forma la edad se convierte en un factor determinante porque no hay confianza y credibilidad en los procesos que desarrollan las jóvenes, con respecto a ello Karen expresó:

"Fui la presidenta de acción comunal más joven de Medellín, era como cierta rivalidad y no solo en mi junta de acción comunal sino en otras partes porque al tema de los jóvenes tampoco se le apuesta, entonces además de ser mujer, era joven, donde la mayoría de juntas de acción comunal están lideradas por gente que lleva 30 años” (E8).

Las intersecciones también se presentan a partir de combinar múltiples condiciones que en sus comunidades significan desigualdades, por ejemplo: ser afro y lesbiana, ser feminista y víctima de la violencia social, ser pobre y campesina, lo que les representa aún mayores obstáculos para participar y liderar procesos. Con relación a lo anterior Julia señaló:

"Si, a una la discriminan por ser mujer, por ser afro, ahora por ser lesbiana, además por ser feminista, además por querer ser radical es que una porta un montón, no de privilegios, sino una no porta el privilegio como mujer negra lesbiana feminista y que sueña con un montón de cosas, no porto yo el privilegio de un montón de cosas” (E4). 


\section{Recomendaciones}

La categoría de sugerencias y recomendaciones se refiere a las propuestas que las mujeres hacen para mejorar sus procesos de participación en la ciudad de Medellín enfocadas a favorecer la equiparación de oportunidades entre hombres y mujeres, apoyo por parte de las instituciones desde los recursos, la creación de espacios de participación y el fortalecimiento de los ya existentes, así como el fomento procesos de formación enfocados a la participación política desde la niñez.

Las recomendaciones dadas por ellas están relacionadas con sus aspiraciones, donde las entrevistadas resaltaron sus ganas de trabajar por sus propias poblaciones. Ese es el caso de las mujeres afrodescendientes, en situación de discapacidad y lesbianas, quienes expresaron su deseo de trabajar en pro de sus comunidades particulares sobre perspectivas relacionadas con la formación, la vinculación a cargos públicos y posicionamiento de lideresas que incidan en los procesos y decisiones a nivel de ciudad.

Figura 5. Categoría: Recomendaciones

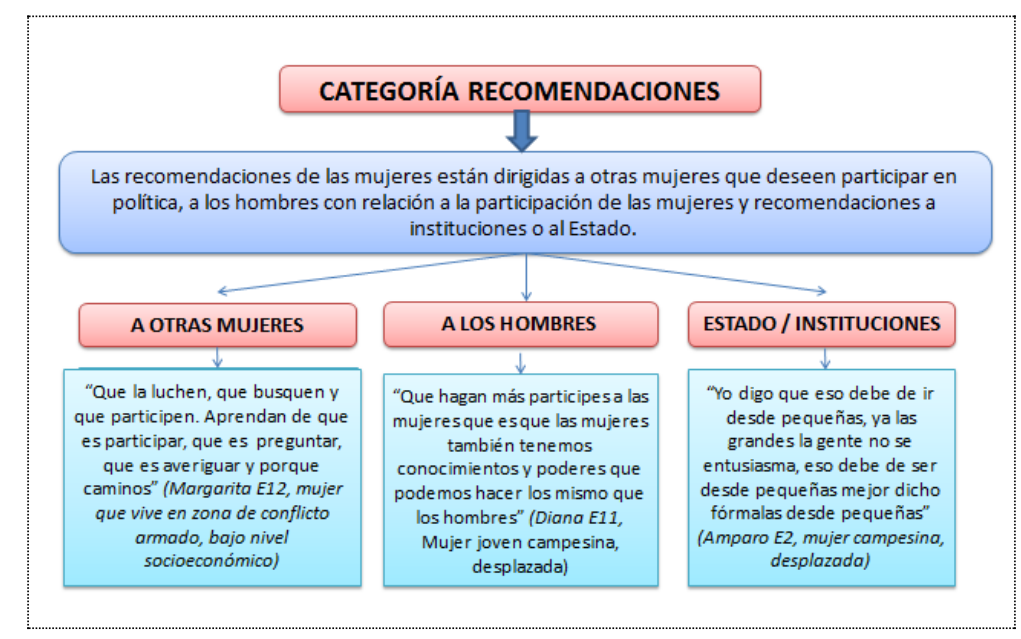

Fuente: Elaboración propia de las autoras basada en los hallazgos de esta investigación

La figura 5 detalla las propuestas que las mujeres hacen para mejorar los procesos de vinculación y permanencia de éstas, en los escenarios de participación política de la ciudad de Medellín: Muchas de estas recomendaciones están dirigidas a otras mujeres para motivar las a superar las barreras personales, a los hombres con quienes comparten estos escenarios, e incluso a las instituciones y el Estado, que se suponen garantes de los derechos civiles y políticos de las mujeres en todo el país. 


\section{Discusión}

De acuerdo con los resultados presentados se ratifica que las mujeres participantes en esta investigación son diversas, pues cada una tiene unas identidades propias vinculadas a su historia o a la pertenencia a un grupo étnico o social, a la construcción incluso de su propia subjetividad, que les sirven para su reconocimiento como lideresas. Sin embargo, ellas dan cuenta de procesos de participación política difíciles en los cuales han tenido que superar dificultades no sólo en relación al género, sino a las intersecciones de éste con las condiciones de diversidad ya señaladas.

El empoderamiento para las mujeres entrevistadas representa un factor fundamental para visibilizarlas como sujetas de derecho y para incidir en los escenarios comunitarios y sociales. Ellas comprenden el empoderamiento como una consecuencia de la participación que les permite incidir y liderar procesos comunitarios. Senso (2011) refiere "El empoderamiento se entiende como un proceso de transformación por el cual el individuo va adquiriendo poder y control para tomar decisiones y alcanzar sus propios objetivos" (Senso, 2011: 12).

La formación es otro factor fundamental para el reconocimiento, ellas han adquirido conocimientos y aprendizajes que les han posibilitado reconocer sus derechos, capacidades, autonomía y liderazgo. De este modo se ha facilitado su gestión comunitaria con los consecuentes logros obtenidos, que a su vez les generan mayores transformaciones personales y sociales. Desde esta perspectiva, para lograr el empoderamiento ha sido clave su formación. Como anota Lagarde (1999):

"Requerimos estar ubicadas en el mundo con conocimiento de causa y por eso la formación de las lideresas es una prioridad junto con la de los procesos de empoderamiento, construir liderazgos es una urgencia, pues las mujeres en el mundo están en situación de emergencia” (Lagarde 1999: 38).

Así pues, promover la formación de mujeres líderes y empoderadas es una necesidad para combatir la cultura patriarcal y los estereotipos sexistas y discriminatorios creados tanto para la esfera pública como privada.

El empoderamiento es la principal herramienta para poder incidir en las comunidades, ganar proyectos y liderar procesos, como una de las claves para luchar contra la cultura patriarcal, ya que las mujeres empoderadas asumen una posición diferente en las relaciones de género que establecen con la familia, la pareja y el contexto, es así como empezaron a visibilizar sus derechos y a resignificar su papel en sus comunidades. León (2000: 14) manifiesta: "Hay que tener en cuenta también que el empoderamiento de las mujeres representa un desafío para las relaciones familiares patriarcales o un desempoderamiento de los hombres o pérdida de la posición privilegiada en que los ha colocado el patriarcado". 
Así mismo, al hablar de empoderamiento Casique (2010) plantea que "este concepto abarca tanto al proceso, como al resultado del proceso por medio del cual las mujeres ganan un mayor control sobre los recursos intelectuales y materiales, y desafían la ideología del patriarcado y la discriminación por género" y participan más activamente.

El concepto de participación política se ha venido transformando a partir de los aportes de la teoría feminista, los cuales proponen comprender la participación en un sentido amplio. Así, las nuevas formas de participación política que las mujeres han venido creando y que no se restringen a representatividad democrática, materializada a través de los diferentes mecanismos de elección constituyen también dispositivos políticos de participación, los cuales tienen una fuerte influencia en la creación y ejecución de políticas públicas en todo el mundo (Elizondo, 2006: 41).

Igualmente, para las mujeres entrevistadas, la participación política está relacionada con la capacidad de opinar, tomar decisiones e incidir en beneficio de la comunidad. Cuando ellas hacen presencia en un escenario de decisión y poder, ya sea de carácter, local, comunal o barrial, las premisas contrahegemónicas que adoptaron para su ejercicio de liderazgo político, les permiten apropiarse de sus escenarios de participación, identificarse con apuestas que contribuyen a transformar las condiciones de vida de su comunidad, es decir promover el empoderamiento social. Todo ello en la búsqueda de generar acciones que han marcado la tendencia política de su participación en beneficio de sus comunidades.

Conviene subrayar, de esta manera, como los liderazgos de estas mujeres, son de mucho trabajo y poco privilegio, lo cual coincide con las palabras de Lagarde (1999: 9), cuando señala que "las mujeres no buscamos convencer ideológicamente con nuestros argumentos y con nuestras propuestas, sino también con acciones. Los liderazgos de las mujeres son liderazgos de acción...".

Respecto a las características del proceso político expresadas por las mujeres consultadas para la presente investigación, se evidencia como participar, es una cualidad propia, que para ellas nació de su ser individual. Sin embargo, al interpretar sus relatos es evidente que ellas se han formado como líderes en contextos estructurales de exclusión, que paradójicamente y pese a lo que se podría esperar, las impulsaron a enarbolar la defensa de sus derechos y los de sus comunidades. De este modo para ellas su participación es una actividad, pues, que parte del deseo y del interés, y que requiere de un alto grado de cumplimiento, voluntad, asertividad, tolerancia y reconocimiento por parte de la comunidad.

Vale la pena, entonces, retomar el concepto de género como una construcción cultural, que permite evidenciar cuando se sitúa al hombre en un rango social mayor al de la mujer que distribuye las ventajas para la participación de forma inequitativa. Como refiere Cobo (2005: 251):

"La inferioridad de las mujeres tiene su génesis en una naturaleza inferior a la masculina. El discurso de la excelencia subraya, sin embargo, la excelencia moral de las mujeres 
respecto de los varones. La paradoja de este discurso es que la excelencia moral de las mujeres se origina precisamente en aquello que las subordina: su asignación al espacio doméstico y su separación del ámbito público-político. Lo significativo de este discurso es que la excelencia se asienta en una normatividad que ha sido el resultado de la jerarquía genérica patriarcal y que se resume en el ejercicio de las tareas de cuidados y en la capacidad de tener sentimientos afectivos y empáticos por parte de las mujeres hacia los otros seres humanos".

Como una forma de control social que prolonga el denominado derecho masculino al castigo (Mojica Rivadeneira, 2005), estas mujeres son sancionadas simbólicamente por transgredir los roles tradicionales de género patriarcales y la excelencia moral femenina. Ello les significa incluso fuertes críticas de forma paradójica por parte de otras mujeres, como parte de la rivalidad entre ellas, promovida por el patriarcado, que como contraparte si alienta los pactos patriarcales entre hombres (Amorós, 1990).

Al analizar como en los procesos de participación las mujeres han tenido diversos obstáculos relacionados con sus condiciones diversas surge "la necesidad de entender la interseccionalidad como una aproximación a la realidad social que permite identificar relaciones de poder y la producción de exclusiones e invisibilización de ciertos grupos sociales" (MISEAL, 2013).

Esta categoría analítica está relacionada directamente con las vivencias de los sujetos en relación al contexto en que se desenvuelven y las relaciones que se establecen con éste desde lo social, cultural y político. Se evidencia a partir de la exclusión y discriminación, pero también en las resistencias que se generan para algunos sujetos o grupos en relación con otros teniendo las mismas o similares condiciones.

Las diferencias en la participación tienen consecuencias que generan procesos de visibilización de los derechos de las mujeres, desde la formación y capacitación, lo que les ha permitido que ellas se resistan, es decir, se miren, se reconozcan, cambien, se piensen, salgan adelante en proyectos donde pueden adquirir sus propios ingresos y transformar sus representaciones, esto es mirar la vida desde otra posición. Por ello, estas mujeres han logrado suscitar credibilidad y confianza en sus comunidades. Sus transformaciones han modificado sus cogniciones y las han impulsado a comunicarlas a nivel social.

\section{Conclusiones}

La participación política y resistencia de las mujeres entrevistadas se da especialmente en espacios locales, en los cuales se evidencia la desigualdad de género pues ellas tienen que 
demostrar más que los hombres líderes, con acciones y proyectos que si pueden incidir en beneficio de sus comunidades, de lo contrario su liderazgo no es validado, ni reconocido.

En relación con la ética del cuidado, la participación de estas mujeres se concentra en tareas de la base, vinculadas a la satisfacción de las necesidades básicas de sus territorios y de las diversas poblaciones que representan en espacios concretos, replicando el rol tradicional femenino en el hogar patriarcal. Paradójicamente este rol ha sido el que las ha motivado a participar para intentar mejorar las condiciones de vida de sus comunidades. En el proceso se han formado y empoderado lo que les ha permitido construirse como seres políticos y empezar a identificar inequidades que antes no visibilizaban.

El reconocimiento es un factor clave en los procesos de participación política de estas mujeres; el cual hace referencia a la legitimación que la comunidad hace de su ejercicio y a la capacidad que tienen ellas para generar bienestar y calidad de vida para la población de su territorio, a través de la gestión de proyectos y procesos concretos.

Las mujeres participantes de la investigación, refieren que las intersecciones de las diversas desigualdades, como la clase social, la discapacidad, la etnia, edad, orientación sexual, entre otras, aumentan los obstáculos de género que han sido determinantes en sus historias de vida por ser mujeres, para incidir en los procesos de participación política. A la vez estos obstáculos las han impelido a resistir y continuar su labor política.

Respecto a los procesos de objetivación y anclaje, las mujeres entrevistadas configuraron sus representaciones sociales de la participación política a partir de las experiencias que vivieron en su socialización temprana, así como en la interacción, comunicación y relación que establecieron con otros sujetos en los diversos escenarios. Es interesante como relatan las transformaciones de sus representaciones de género.

La representación social de la participación política para este grupo de mujeres es la posibilidad de incidir en los diferentes espacios sociales, comunitarios y políticos, en los cuales pueden opinar para tomar decisiones y gestionar proyectos y acciones en beneficio de sus comunidades, además hace referencia a la posibilidad de elegir y ser elegidas.

El empoderamiento de estas mujeres, en sus procesos de participación política, es crucial para su desarrollo político y social, pues les facilita perder el miedo para opinar, enfrentarse a los diferentes escenarios y luchar por los proyectos de sus comunidades, es decir, les permite reconocer sus derechos y resistir.

Las familias constituyen un eje central en los procesos de participación política de estas mujeres, ya que representan un importante facilitador o un gran obstaculizador del ejercicio de liderazgo de las mismas; porque implica que las mujeres cumplan con todos los compromisos vinculados con su triple carga como madres, trabajadoras y líderes, para ser autorizadas por así decirlo, por sus familias y no tener que desertar de su proceso de liderazgo o de sus relaciones afectivas de pareja.

A pesar de su trabajo en pro de la comunidad y de sus logros, estas mujeres son sancionadas en diferentes formas por sus comunidades y por sus familiares hombres por 
transgredir los roles tradicionales de género patriarcales y la excelencia moral femenina. El liderazgo se convirtió para las mujeres entrevistadas en su ocupación y esencia principal, aunque hayan tenido que realizar diversos "sacrificios" y resistencias para poder ejercer su rol político de lideresas.

Lograr la equidad de género y la inclusión de las mujeres en el ejercicio pleno de la participación política es un hecho relevante para Colombia en el siglo XXI, su representatividad y paridad constituyen elementos fundamentales para contrarrestar las barreras y las representaciones culturales construidas históricamente y que aún se esgrimen para intentar marginalizarlas de los escenarios políticos. Luchar por la igualdad de condiciones se hace necesario, pues las mujeres no solo han estado excluidas por su condición de género sino, además, por otras condiciones sociales, económicas, étnicas, de orientación sexual o físicas, situación que se ha ido transformando a partir de los procesos de resistencia y movilización de ellas mismas.

\section{BIBLIOGRAFÍA}

- Amorós, Celia (1990): Violencia contra las mujeres y pactos patriarcales. Madrid: Pablo Iglesias.

- Andrade, Daniela (2006): "Políticas Públicas con Perspectiva de Género que potencien la Participación de la Mujer en el Poder y la Toma de Decisiones en América Latina y el Caribe", [en línea] Disponible en: http://www.ts.ucr.ac.cr/binarios/pela/pl-000294.pdf [25/01/2016].

- Casique, Irene (2010): "Factores de empoderamiento y protección de las mujeres contra la violencia”. En: Revista Mexicana de Sociología, no. 1, pp. 37-71.

- Cobo, Rosa (2005): "El género en las ciencias sociales". En: Revista Cuadernos de trabajo social, vol. 18, pp. 249-258.

- Colombia, Congreso (2000): “Ley 581 por la cual se reglamenta la adecuada y efectiva participación de la mujer en los niveles decisorios de las diferentes ramas y órganos del poder público, de conformidad con los artículos 13, 40 y 43 de la Constitución Nacional y se dictan otras disposiciones". En: Diario Oficial, n ${ }^{\circ} 44.026$.

- Corbin y Strauss (2002): Bases de la investigación cualitativa. Técnicas y procedimientos para desarrollar la teoría fundamentada. Colombia: Contus Editorial Universidad de Antioquia.

- Creswell, John W. (2007): Qualitative Inquiry and Research Design: Choosing Among Five Traditions. California: Sage

- Elizondo, Arantxa (2006): “La participación política de las mujeres en Euskadi”. En: Revista Estudios de Género de la Universidad de Alicante, nº. 3, pp. 75-88.

- Expósito, Carmen (2012): “¿Qué es eso de la interseccionalidad?: Aproximación al tratamiento de la diversidad desde la perspectiva de género en España". En: Revista Investigaciones Feministas, vol. 3, pp. 203-222. 
- Guba, Egon (2008) [1989]: “Criterios de credibilidad en la investigación naturalista”. En: José Gimeno y Angel Pérez: La enseñanza: su teoría y su práctica. Madrid: Akal.

- Idrovo, Sandra. (2006): "Las políticas de conciliación trabajo-familia en las empresas colombianas". En: Estudios Gerenciales, vol. 22, nº 100, pp. 49-70.

- Jodelet, Denise (1984): "La representación social: fenómenos, concepto y teoría". En: Serge Moscovici: Psicología social, II. Pensamiento y vida social. Psicología social y problemas sociales, $\mathrm{n}^{\circ}$. 2, Barcelona: Paidós, pp. 481-494.

- Jurado, Juan Carlos (2003): "Problemas y tendencias contemporáneas de la vida familiar y urbana en Medellín”. En: Revista Historia Crítica, nº 25, pp. 165-182.

- Lagarde, Marcela (1999): “Claves feministas para liderazgos entrañables”, [en línea] Disponible en: http://sidoc.puntos.org.ni/publicacionesptos/documentos/claves-paraiderazgos.pdf [25/01/2016].

- León, Magdalena (2000): "Empoderamiento: relaciones de las mujeres con el poder". En: Revista Estudios Feministas, vol. 8, n . 2, pp. 191-207.

- Luna, Lola (2002): "La historia feminista del género y la cuestión del sujeto", [en línea] Disponible en: http://www.lolagluna.com/publicaciones/articulos/Lahistoria.pdf [25/01/2016].

- MISEAL (2013): "La interseccionalidad en debate", [en línea] Disponible en: http://www.upla.cl/inclusion/wpcontent/uploads/2015/04/Interseccionalidadendebate_misealwe b-1.pdf [25/01/2016].

- Mojica Rivadeneira, Maria Teresa (2005): "El Derecho masculino de castigo en la Colonia". En: René Salinas Meza y María Teresa Mojica Rivadeneira: Conductas ilícitas y derecho de castigo durante la colonia. Los casos de Chile y Colombia, 87-198. Bogotá: Universidad Externado de Colombia.

- Puyana, Yolanda y Mosquera Claudia (2005): “Traer hijos o hijas al mundo: significados culturales de la paternidad y la maternidad". En: Revista Latinoamericana de Ciencias sociales niñez y juventud, vol. 3, no. 2, Manizales July/Dec., pp. 2-21

- Tello, Flavia (2009): "La participación política de las mujeres en los gobiernos locales latinoamericanos: Barreras y desafíos para una efectiva democracia de género", [en línea] Disponible en: http://www.americalatinagenera.org/es/index.php?option=com_content\&task=view\&id $=905 \& p$ $\mathrm{ub} \_\mathrm{id}=1707 \& \mathrm{ml}=1 \& \mathrm{mlt}=$ system\&tmpl=component $[25 / 01 / 2016]$.

- Touraine, Alain (1993). Crítica de la modernidad. Traducido por Mauro Armiño. Madrid: Temas de Hoy. 